\title{
Perturbation Analysis for Elastic Problems with Slight-Rough Straight Boundary*
}

\author{
Masayuki ARAI** \\ **Materials Science Research Lab., Central Research Institute of Electric Power Industry, \\ 2-11-1 Iwado-kita, Komae-shi, Tokyo 201-8511, Japan \\ E-mail: marai@criepi.denken.or.jp
}

\begin{abstract}
In this paper, first-order solutions for elastic problems with a slight-rough straight boundary shape as an arbitrary shape function $y=g(x)$ are obtained based on a perturbation theory. The first-order solutions obtained here are valid to $\mathrm{O}(\varepsilon)$ order where $\varepsilon=A / \lambda<<1$; A is the amplitude and $\lambda$ is the characteristic wavelength in the shape function. Firstly, a general solution for an elastic problem with a straight boundary disturbed slightly by the shape function, $y=g(x)$, is shown. In this study, cosine as a typical periodic function and an exponential function are considered as the shape function. As an application of the general solution, the first-order solutions for semi-infinite elastic media subjected to point load and concentrated moment are shown, and these also are compared with the results obtained by finite element analysis.
\end{abstract}

Key words: Elastic Problem, Perturbation Analysis, Slight-Rough Boundary Problem

\section{Introduction}

In this study, an elastic problem with a slight rough boundary is considered. This elastic problem is important in the following engineering fields. Free surface in homogeneous media and boundary of adherence in a bonded joint have a somewhat rough boundary. Those rough boundaries affect the strength or life of mechanical components. For instance, it is well known that roughness of the surface leads to reduction of fatigue life ${ }^{(1)}$, and also the stress concentration at the edge of the punch is reduced when a flat-rigid punch is indented on the surface with roughness ${ }^{(2)}$. Otherwise, in an epitaxially grown thin film, residual stress formed during the epitaxial process leads to a wavy free surface ${ }^{(3)}$. Thus, it is quite important to clarify the effect of those wavy boundaries on stress fields in elastic media.

There are some papers related to those problems. Gao ${ }^{(4)}$ has obtained stress concentration factors at the edge of a hole in infinite plate when a circle-hole shape is changed slightly to an elliptical one and to a smooth polygon one. He has solved for first-approximation solutions based on the energy principle method proposed by Rice ${ }^{(5)}$. He (6) has also approached the same problems using Muskhelishvilli's complex stress function expression. Wang et al. ${ }^{(7)}$ have obtained an exact solution for the problem of semi-infinite elastic media with a cosine-wavy free surface subjected to a point load. The solution has been derived by an Airy stress function method, in which the stress component expression ${ }^{(8)}$ in Cartesian coordinates is transformed to curvilinear coordinates. Srolovitz ${ }^{(3)}$ has also solved for semi-infinite media with sine-wavy free surface subjected to a far-filed tensile loading, by using Fourier series expansion in an Airy stress function. Some solutions for a punch problem with periodic rough surface are summarized in Jonson's book ${ }^{(2)}$. Villaggio ${ }^{(9)}$ 
has discussed the effect of a wavy joint boundary on a stress field, using a potential theory based on a Boussinesq-Papkovic-Neuber potential function. Although some exact or approximate solutions have been solved, the forms of solutions obtained are quite complicated, and it is difficult to directly apply these to engineering problems.

In this study, perturbation theory is applied to a two-dimensional elastic problem with a slight-rough straight boundary. First, equilibrium equations for displacement in Cartesian coordinates $(x, y)$ are translated to in curvilinear coordinates $(\xi, \eta)$, considering the shape function $y=g(x)$ to express the rough boundary. Next, general first-order solutions for displacement and stress components are obtained through a perturbation scheme including the small parameter $\varepsilon=A / \lambda<<$, where $A$ is amplitude and $\lambda$ is the characteristic wavelength in the shape function. That is, this solution is derived with the assumption that the amplitude of the shape function is small when compared with the wavelength, and is valid to $O(\varepsilon)$ order.

As an application of the general first-order solution obtained here, the cosine as a typical periodic function and exponential function are considered as the shape functions. The solutions for semi-infinite elastic media subjected to point load and concentrated moment are shown as examples, and these also are compared with the results obtained by finite element analysis.

\section{Basic Equations for Problem}

In this study, two-dimensional linear elastic problems bounded with slight-wavy straight boundaries laid along the $x$-axis are considered. It is assumed that the slight-wavy boundary is given by the following shape function:

$$
y=g(x) \quad-\infty<x<\infty
$$

For a linear elastic, homogeneous and isotropic media in absent of body forces, the equilibrium equations are:

$$
(\kappa-1) \nabla^{2} \vec{u}+2 \nabla e=0
$$

where $\vec{u}$ is the displacement vector with components $(u, v)$ and $\kappa$ is Kolosov's constants; $(3-v) /(1+v)$ for plane stress and $(3-4 v)$ for plane strain, dilatation $e$ is given by,

$$
e=\frac{\partial u}{\partial y}+\frac{\partial}{\partial x}=\nabla \cdot \vec{u}
$$

, $\nabla$ is the gradient operator, and $\nabla^{2}=\nabla \cdot \nabla$ is the Laplace operator.

Stress components are,

$$
\begin{aligned}
& \sigma_{x x}=2 \mu\left[\varepsilon_{x x}-\frac{1}{2}\left(\frac{\kappa-3}{\kappa-1}\right) e\right] \\
& \sigma_{y y}=2 \mu\left[\varepsilon_{y y}-\frac{1}{2}\left(\frac{\kappa-3}{\kappa-1}\right) e\right] \\
& \sigma_{x y}=\mu \gamma_{x y}
\end{aligned}
$$

where $\mu$ is the shear modulus of the elastic media and $\varepsilon_{x x}, \varepsilon_{y y}, \gamma_{x y}$ are strain components as follows;

$$
\varepsilon_{x x}=\frac{\partial u}{\partial x}, \varepsilon_{y y}=\frac{\partial}{\partial y}, \gamma_{x y}=\frac{\partial u}{\partial y}+\frac{\partial}{\partial x}
$$

in Cartesian coordinates $(x, y)$, respectively.

For applying perturbation theory to this problem, we introduce the following 
transformation from Cartesian $(x, y)$ to curvilinear coordinates $(\xi, \eta)$ :

$$
\begin{aligned}
& \xi=x \\
& \eta=y-g(x)
\end{aligned}
$$

where the above relation describes the wavy boundary at $\eta=0$.

The differential chain rule between $(x, y)$ and $(\xi, \eta)$ is given in the following form:

$$
\left(\begin{array}{ll}
\frac{\partial \xi}{\partial x} & \frac{\partial \xi}{\partial y} \\
\frac{\partial \eta}{\partial x} & \frac{\partial \eta}{\partial y}
\end{array}\right)=\frac{1}{J}\left(\begin{array}{cc}
\frac{\partial y}{\partial \eta} & -\frac{\partial x}{\partial \eta} \\
-\frac{\partial y}{\partial \xi} & \frac{\partial x}{\partial \xi}
\end{array}\right)
$$

where $J$ is,

$$
J \equiv\left|\begin{array}{ll}
\frac{\partial x}{\partial \xi} & \frac{\partial x}{\partial \eta} \\
\frac{\partial y}{\partial \xi} & \frac{\partial y}{\partial \eta}
\end{array}\right|(\neq 0)
$$

The transformation relation (6) is also rewritten as,

$$
\begin{aligned}
& x=\xi \\
& y=\eta+g(\xi)
\end{aligned}
$$

Substituting the relation (9) into (8) and then (7), $J=1$ and also,

$$
\left(\begin{array}{ll}
\frac{\partial \xi}{\partial x} & \frac{\partial \xi}{\partial y} \\
\frac{\partial \eta}{\partial x} & \frac{\partial \eta}{\partial y}
\end{array}\right)=\left(\begin{array}{cc}
1 & 0 \\
-\frac{d g(\xi)}{d \xi} & 1
\end{array}\right)
$$

Now, consider transformation coordinates for the gradient and Laplace operators using the chain rule relation (10). The gradient operator $\nabla$ becomes

$$
\begin{aligned}
& \nabla_{x}=\frac{\partial \xi}{\partial x} \frac{\partial}{\partial \xi}+\frac{\partial \eta}{\partial x} \frac{\partial}{\partial \eta}=\frac{\partial}{\partial \xi}-\left(\frac{d g(\xi)}{d \xi}\right) \cdot \frac{\partial}{\partial \eta} \\
& \nabla_{y}=\frac{\partial \xi}{\partial y} \frac{\partial}{\partial \xi}+\frac{\partial \eta}{\partial y} \frac{\partial}{\partial \eta}=\frac{\partial}{\partial \eta}
\end{aligned}
$$

The Laplace operator $\nabla^{2}$ :

$$
\nabla^{2}=\frac{\partial^{2}}{\partial x^{2}}+\frac{\partial^{2}}{\partial y^{2}}=\hat{\nabla}^{2}-\left[\frac{d^{2} g(\xi)}{d \xi^{2}}+2\left(\frac{d g(\xi)}{d \xi}\right) \frac{\partial}{\partial \xi}+\left(\frac{d g(\xi)}{d \xi}\right)^{2} \frac{\partial}{\partial \eta}\right] \frac{\partial}{\partial \eta}
$$

where the new operator $\hat{\nabla}^{2}$ is defined by,

$$
\hat{\nabla}^{2}=\frac{\partial^{2}}{\partial \xi^{2}}+\frac{\partial^{2}}{\partial \eta^{2}}
$$

Substituting the operators (11) and (12) into the equilibrium equation (2), the equations suited to this problem are obtained in the following forms:

$$
\begin{aligned}
& (\kappa-1) \hat{\nabla}^{2} u+2 \frac{\partial}{\partial \xi} \hat{e}=\frac{\partial}{\partial \eta}\left[(\kappa+1) \frac{d^{2} g}{d \xi^{2}} \cdot u+2(\kappa+1) \frac{d g}{d \xi} \frac{\partial u}{\partial \xi}+2 \frac{d g}{d \xi} \frac{\partial}{\partial \eta}+(\kappa-3)\left(\frac{d g}{d \xi}\right)^{2} \frac{\partial u}{\partial \eta}\right] \\
& (\kappa-1) \hat{\nabla}^{2} v+2 \frac{\partial}{\partial \eta} \hat{e}=\frac{\partial}{\partial \eta}\left[(\kappa-1) \frac{d^{2} g}{d \xi^{2}} \cdot v+2(\kappa-1) \frac{d g}{d \xi} \frac{\partial}{\partial \xi}+2 \frac{d g}{d \xi} \frac{\partial u}{\partial \eta}+(\kappa-1)\left(\frac{d g}{d \xi}\right)^{2} \frac{\partial}{\partial \eta}\right]
\end{aligned}
$$


where the displacement components $u=u(\xi, \eta)$ and $v=v(\xi, \eta)$, and the dilatation $\hat{e}$ is

$$
\hat{e}=\frac{\partial u}{\partial \xi}+\frac{\partial}{\partial \eta}
$$

Associated stress components can be also translated into the following form in the same way.

$$
\begin{aligned}
& \sigma_{x x}(\xi, \eta)=2 \mu\left[\left(\frac{\partial u}{\partial \xi}-\frac{1}{2}\left(\frac{\kappa-3}{\kappa-1}\right) \hat{e}\right)-\frac{1}{2}\left(\frac{\kappa+1}{\kappa-1}\right) \frac{d g}{d \xi} \frac{\partial u}{\partial \eta}\right] \\
& \sigma_{y y}(\xi, \eta)=2 \mu\left[\left(\frac{\partial}{\partial \eta}-\frac{1}{2}\left(\frac{\kappa-3}{\kappa-1}\right) \hat{e}\right)+\frac{1}{2}\left(\frac{\kappa-3}{\kappa-1}\right) \frac{d g}{d \xi} \frac{\partial u}{\partial \eta}\right] \\
& \sigma_{x y}(\xi, \eta)=\mu\left[\left(\frac{\partial u}{\partial \eta}+\frac{\partial}{\partial \xi}\right)-\frac{d g}{d \xi} \frac{\partial}{\partial \eta}\right]
\end{aligned}
$$

\section{General First-order Solutions from Perturbation Analysis}

In this study, a periodic shape function, the cosine, and an exponential-type shape function are considered as the shape function.

\subsection{Case of cosine shape function}

Here, the cosine shape function is given as the following representation:

$$
y=A \cos \left(\frac{2 \pi}{\lambda} x\right)
$$

where $A$ is the amplitude and $\lambda$ is the wavelength of the wavy boundary. If taking $A \rightarrow 0$ or $\lambda \rightarrow \infty$, the wavy boundary becomes the straight boundary.

The transformation relation in this problem becomes,

$$
\begin{aligned}
& x=\xi \\
& y=\eta+A \cos \left(\frac{2 \pi}{\lambda} \xi\right)
\end{aligned}
$$

Substituting (18) into (14), we have

$$
\begin{array}{r}
(\kappa-1) \hat{\nabla}^{2} u+2 \frac{\partial}{\partial \xi} \hat{e}=\frac{\partial}{\partial \eta}\left[-4 \pi^{2}(\kappa+1) \frac{A}{\lambda^{2}} \cos \left(\frac{2 \pi}{\lambda} \xi\right) \cdot u-4 \pi(\kappa+1) \frac{A}{\lambda} \sin \left(\frac{2 \pi}{\lambda} \xi\right) \frac{\partial u}{\partial \xi}\right. \\
\left.-4 \pi \frac{A}{\lambda} \sin \left(\frac{2 \pi}{\lambda} \xi\right) \frac{\partial}{\partial \eta}+4 \pi^{2}(\kappa-3)\left(\frac{A}{\lambda}\right)^{2} \sin ^{2}\left(\frac{2 \pi}{\lambda} \xi\right) \frac{\partial u}{\partial \eta}\right] \\
(\kappa-1) \hat{\nabla}^{2} v+2 \frac{\partial}{\partial \eta} \hat{e}=\frac{\partial}{\partial \eta}\left[-4 \pi^{2}(\kappa-1) \frac{A}{\lambda^{2}} \cos \left(\frac{2 \pi}{\lambda} \xi\right) \cdot v-4 \pi(\kappa-1) \frac{A}{\lambda} \sin \left(\frac{2 \pi}{\lambda} \xi\right) \frac{\partial}{\partial \xi}\right. \\
\left.-4 \pi \frac{A}{\lambda} \sin \left(\frac{2 \pi}{\lambda} \xi\right) \frac{\partial u}{\partial \eta}+4 \pi^{2}(\kappa-1)\left(\frac{A}{\lambda}\right)^{2} \sin ^{2}\left(\frac{2 \pi}{\lambda} \xi\right) \frac{\partial v}{\partial \eta}\right]
\end{array}
$$

Here, introducing the normalized parameters $\tilde{\xi}=\xi / A, \tilde{\eta}=\eta / A$ and $\varepsilon=A / \lambda$, the equations (19) can be reduced to the forms: 


$$
\begin{array}{r}
(\kappa-1) \tilde{\hat{\nabla}}^{2} u+2 \frac{\partial}{\partial \tilde{\xi}} \widetilde{\hat{e}}=\frac{\partial}{\partial \widetilde{\eta}}\left[-4 \pi^{2}(\kappa+1) \varepsilon^{2} \cos (2 \pi \varepsilon \tilde{\xi}) \cdot u-4 \pi(\kappa+1) \varepsilon \sin (2 \pi \varepsilon \tilde{\xi}) \frac{\partial u}{\partial \widetilde{\xi}}\right. \\
\left.-4 \pi \varepsilon \sin (2 \pi \varepsilon \tilde{\xi}) \frac{\partial}{\partial \widetilde{\eta}}+4 \pi^{2}(\kappa-3) \varepsilon^{2} \sin ^{2}(2 \pi \varepsilon \tilde{\xi}) \frac{\partial u}{\partial \widetilde{\eta}}\right] \\
(\kappa-1) \widetilde{\hat{\nabla}}^{2} v+2 \frac{\partial}{\partial \tilde{\eta}} \hat{e}=\frac{\partial}{\partial \widetilde{\eta}}\left[-4 \pi^{2}(\kappa-1) \varepsilon^{2} \cos (2 \pi \varepsilon \tilde{\xi}) \cdot v-4 \pi(\kappa-1) \varepsilon \sin (2 \pi \varepsilon \tilde{\xi}) \frac{\partial}{\partial \tilde{\xi}}\right. \\
\left.-4 \pi \varepsilon \sin (2 \pi \varepsilon \tilde{\xi}) \frac{\partial u}{\partial \widetilde{\eta}}+4 \pi^{2}(\kappa-1) \varepsilon^{2} \sin ^{2}(2 \pi \varepsilon \tilde{\xi}) \frac{\partial}{\partial \widetilde{\eta}}\right]
\end{array}
$$

where

$$
\tilde{\hat{\nabla}}^{2}=\frac{\partial^{2}}{\partial \widetilde{\xi}^{2}}+\frac{\partial^{2}}{\partial \widetilde{\eta}^{2}}, \quad \tilde{\hat{e}}=\frac{\partial u}{\partial \widetilde{\xi}}+\frac{\partial}{\partial \widetilde{\eta}}
$$

Furthermore, if we let $\widetilde{\xi}_{1} \equiv \varepsilon \tilde{\xi}, \quad \tilde{\eta}_{1} \equiv \varepsilon \tilde{\eta}$, the equations (20) are reduced further,

$$
\begin{array}{r}
(\kappa-1) \tilde{\hat{\nabla}}_{1}^{2} u+2 \frac{\partial}{\partial \widetilde{\xi}_{1}} \widetilde{\hat{1}}_{1}=\frac{\partial}{\partial \widetilde{\eta}_{1}}\left[-4 \pi^{2}(\kappa+1) \varepsilon \cos \left(2 \pi \widetilde{\xi}_{1}\right) \cdot u-4 \pi(\kappa+1) \varepsilon \sin \left(2 \pi \widetilde{\xi}_{1}\right) \frac{\partial u}{\partial \widetilde{\xi}_{1}}\right. \\
\left.-4 \pi \varepsilon \sin \left(2 \pi \widetilde{\xi}_{1}\right) \frac{\partial}{\partial \widetilde{\eta}_{1}}+4 \pi^{2}(\kappa-3) \varepsilon^{2} \sin ^{2}\left(2 \pi \widetilde{\xi}_{1}\right) \frac{\partial u}{\partial \widetilde{\eta}_{1}}\right] \\
(\kappa-1) \hat{\nabla}_{1}^{2} v+2 \frac{\partial}{\partial \widetilde{\eta}_{1}} \widetilde{\hat{e}}_{1}=\frac{\partial}{\partial \widetilde{\eta}_{1}}\left[-4 \pi^{2}(\kappa-1) \varepsilon \cos \left(2 \pi \widetilde{\xi}_{1}\right) \cdot v-4 \pi(\kappa-1) \varepsilon \sin \left(2 \pi \widetilde{\xi}_{1}\right) \frac{\partial}{\partial \widetilde{\xi}_{1}}\right. \\
\left.-4 \pi \varepsilon \sin \left(2 \pi \widetilde{\xi}_{1}\right) \frac{\partial u}{\partial \widetilde{\eta}_{1}}+4 \pi^{2}(\kappa-1) \varepsilon^{2} \sin ^{2}\left(2 \pi \widetilde{\xi}_{1}\right) \frac{\partial}{\partial \widetilde{\eta}_{1}}\right]
\end{array}
$$

where

$$
\tilde{\hat{\nabla}}_{1}^{2}=\frac{\partial^{2}}{\partial \widetilde{\xi}_{1}^{2}}+\frac{\partial^{2}}{\partial \widetilde{\eta}_{1}^{2}}, \quad \widetilde{\hat{e}}_{1}=\frac{\partial u}{\partial \widetilde{\xi}_{1}}+\frac{\partial}{\partial \widetilde{\eta}_{1}}
$$

Here, if it is assumed that $\varepsilon=A / \lambda<<1, \varepsilon$ can be regarded as the perturbation parameter. Thus, the displacement components can be taken as the following linear series expansion around the perturbation parameter:

$$
\begin{aligned}
& u\left(\widetilde{\xi}_{1}, \widetilde{\eta}_{1}\right) \approx u^{0}\left(\widetilde{\xi}_{1}, \widetilde{\eta}_{1}\right)+\varepsilon u^{1}\left(\widetilde{\xi}_{1}, \widetilde{\eta}_{1}\right)+\varepsilon^{2} u^{2}\left(\widetilde{\xi}_{1}, \widetilde{\eta}_{1}\right)+\cdots \cdots \\
& v\left(\widetilde{\xi}_{1}, \widetilde{\eta}_{1}\right) \approx v^{0}\left(\widetilde{\xi}_{1}, \widetilde{\eta}_{1}\right)+\varepsilon v^{1}\left(\widetilde{\xi}_{1}, \widetilde{\eta}_{1}\right)+\varepsilon^{2} v^{2}\left(\widetilde{\xi}_{1}, \widetilde{\eta}_{1}\right)+\cdots \cdots
\end{aligned}
$$

Substituting (22) into (21) and comparing with each factor for $\varepsilon^{n}$ order $(n=1,2,3 \ldots$.$) ,$ the following differential equations are derived.

(i) $O(1)$ order:

$$
\begin{aligned}
& (\kappa-1) \widetilde{\hat{\nabla}}_{1}^{2} u^{0}+2 \frac{\partial}{\partial \widetilde{\xi}_{1}} \widetilde{\hat{e}}_{1}^{0}=0 \\
& (\kappa-1) \hat{\nabla}_{1}^{2} v^{0}+2 \frac{\partial}{\partial \widetilde{\eta}_{1}} \widetilde{\hat{e}}_{1}^{0}=0
\end{aligned}
$$

(ii) $O(\varepsilon)$ order:

$$
\begin{aligned}
& (\kappa-1) \widetilde{\hat{\nabla}}_{1}^{2} u^{1}+2 \frac{\partial}{\partial \widetilde{\xi}_{1}} \widetilde{\hat{e}}_{1}^{1}=\frac{\partial}{\partial \widetilde{\eta}_{1}}\left[-4 \pi^{2}(\kappa+1) \cos \left(2 \pi \widetilde{\xi}_{1}\right) \cdot u^{0}-4 \pi(\kappa+1) \sin \left(2 \pi \widetilde{\xi_{1}}\right) \frac{\partial u^{0}}{\partial \widetilde{\xi}_{1}}-4 \pi \sin \left(2 \pi \widetilde{\xi}_{1}\right) \frac{\partial^{0}}{\partial \widetilde{\eta}_{1}}\right] \\
& (\kappa-1) \hat{\nabla}_{1}^{2} v^{1}+2 \frac{\partial}{\partial \widetilde{\eta}_{1}} \widetilde{\hat{e}}_{1}^{1}=\frac{\partial}{\partial \widetilde{\eta}_{1}}\left[-4 \pi^{2}(\kappa-1) \cos \left(2 \pi \widetilde{\xi}_{1}\right) \cdot v^{0}-4 \pi(\kappa-1) \sin \left(2 \pi \widetilde{\xi_{1}}\right) \frac{\partial^{0}}{\partial \widetilde{\xi}_{1}}-4 \pi \varepsilon \sin \left(2 \pi \widetilde{\xi_{1}}\right) \frac{\partial u^{0}}{\partial \widetilde{\eta}_{1}}\right]
\end{aligned}
$$

and so on. The first equation corresponds to the non-perturbation term - that is, to the original term for the straight-boundary problem — and the second one includes a slightly changed term by first-order perturbation. 
We note that equations (24) satisfy the following relations (it is easy to check this by substituting (25) into (24)):

$$
\begin{aligned}
& u^{1}\left(\widetilde{\xi}_{1}, \tilde{\eta}_{1}\right)=\frac{\partial}{\partial \widetilde{\eta}_{1}} u^{0}\left(\widetilde{\xi}_{1}, \tilde{\eta}_{1}\right) \cdot \cos \left(2 \pi \widetilde{\xi}_{1}\right) \\
& v^{1}\left(\widetilde{\xi}_{1}, \tilde{\eta}_{1}\right)=\frac{\partial}{\partial \widetilde{\eta}_{1}} v^{0}\left(\widetilde{\xi}_{1}, \tilde{\eta}_{1}\right) \cdot \cos \left(2 \pi \widetilde{\xi}_{1}\right)
\end{aligned}
$$

Finally, a general first-order solution for a cosine-wavy boundary problem can be given in the forms:

$$
\begin{aligned}
& u\left(\widetilde{\xi}_{1}, \tilde{\eta}_{1}\right) \approx u^{0}\left(\widetilde{\xi}_{1}, \tilde{\eta}_{1}\right)+\varepsilon \frac{\partial}{\partial \widetilde{\eta}_{1}} u^{0}\left(\widetilde{\xi}_{1}, \tilde{\eta}_{1}\right) \cdot \cos \left(2 \pi \tilde{\xi}_{1}\right) \\
& v\left(\widetilde{\xi}_{1}, \tilde{\eta}_{1}\right) \approx v^{0}\left(\widetilde{\xi}_{1}, \tilde{\eta}_{1}\right)+\varepsilon \frac{\partial}{\partial \widetilde{\eta}_{1}} v^{0}\left(\widetilde{\xi}_{1}, \tilde{\eta}_{1}\right) \cdot \cos \left(2 \pi \widetilde{\xi}_{1}\right)
\end{aligned}
$$

, and we have following solution by returning each parameter: $\varepsilon, \xi, \eta$ and so on.

$$
\begin{aligned}
& u(\xi, \eta) \approx u^{0}(\xi, \eta)+\frac{\partial}{\partial \eta} u^{0}(\xi, \eta) \cdot A \cos \left(\frac{2 \pi}{\lambda} \xi\right) \\
& v(\xi, \eta) \approx v^{0}(\xi, \eta)+\frac{\partial}{\partial \eta} v^{0}(\xi, \eta) \cdot A \cos \left(\frac{2 \pi}{\lambda} \xi\right)
\end{aligned}
$$

Substituting (27) into (16), the associated stress components become,

$$
\begin{aligned}
& \sigma_{x x}(\xi, \eta) \approx \sigma_{x x}^{0}(\xi, \eta)+\frac{\mu}{\kappa-1}\left\{(\kappa+1) \frac{\partial^{2} u^{0}}{\partial \xi \partial \eta}-(\kappa-3) \frac{\partial^{2} v^{0}}{\partial \eta^{2}}\right\} A \cos \left(\frac{2 \pi}{\lambda} \xi\right) \\
& \sigma_{y y}(\xi, \eta) \approx \sigma_{y y}^{0}(\xi, \eta)-\frac{\mu}{\kappa-1}\left\{(\kappa-3) \frac{\partial^{2} u^{0}}{\partial \xi \partial \eta}-(\kappa+1) \frac{\partial^{2} v^{0}}{\partial \eta^{2}}\right\} A \cos \left(\frac{2 \pi}{\lambda} \xi\right) \\
& \sigma_{x y}(\xi, \eta) \approx \sigma_{x y}^{0}(\xi, \eta)+\mu\left\{\frac{\partial^{2} u^{0}}{\partial \eta^{2}}+\frac{\partial^{2} v^{0}}{\partial \xi \partial \eta}\right\} A \cos \left(\frac{2 \pi}{\lambda} \xi\right)
\end{aligned}
$$

\subsection{Case of exponential-type shape function}

Next, the following shape function is considered.

$$
y=A \exp \left\{-\left(\frac{x}{h}\right)^{2}\right\}
$$

Here, if taking $A \rightarrow 0$ and $h \rightarrow \infty$, the wavy boundary becomes the straight boundary.

Transformation (9) can be set,

$$
\begin{aligned}
& x=\xi \\
& y=\eta+A \exp \left(-\left(\frac{\xi}{h}\right)^{2}\right)
\end{aligned}
$$

First-order solutions for an exponential-type shape function problem can be also obtained, using the same scheme as in the case of the cosine shape function problem, as follows.

1) The displacement solutions are, 


$$
\begin{aligned}
& u(\xi, \eta) \approx u^{0}(\xi, \eta)+\frac{\partial}{\partial \eta} u^{0}(\xi, \eta) \cdot A \exp \left\{-\left(\frac{\xi}{h}\right)^{2}\right\} \\
& v(\xi, \eta) \approx v^{0}(\xi, \eta)+\frac{\partial}{\partial \eta} v^{0}(\xi, \eta) \cdot A \exp \left\{-\left(\frac{\xi}{h}\right)^{2}\right\}
\end{aligned}
$$

, and 2) the stress components are also,

$$
\begin{aligned}
& \sigma_{x x}(\xi, \eta) \approx \sigma_{x x}^{0}(\xi, \eta)+\frac{\mu}{\kappa-1}\left\{(\kappa+1) \frac{\partial^{2} u^{0}}{\partial \xi \partial \eta}-(\kappa-3) \frac{\partial^{2} v^{0}}{\partial \eta^{2}}\right\} A \exp \left\{-\left(\frac{\xi}{h}\right)^{2}\right\} \\
& \sigma_{y y}(\xi, \eta) \approx \sigma_{y y}^{0}(\xi, \eta)-\frac{\mu}{\kappa-1}\left\{(\kappa-3) \frac{\partial^{2} u^{0}}{\partial \xi \partial \eta}-(\kappa+1) \frac{\partial^{2} v^{0}}{\partial \eta^{2}}\right\} A \exp \left\{-\left(\frac{\xi}{h}\right)^{2}\right\} \\
& \sigma_{x y}(\xi, \eta) \approx \sigma_{x y}^{0}(\xi, \eta)+\mu\left\{\frac{\partial^{2} u^{0}}{\partial \eta^{2}}+\frac{\partial^{2} v^{0}}{\partial \xi \partial \eta}\right\} A \exp \left\{-\left(\frac{\xi}{h}\right)^{2}\right\}
\end{aligned}
$$

\section{Application of General First-Order Solution}

As applications of the general first-order solution obtained above, two kinds of elastic problems are considered: a semi-infinite elastic problem subjected to point load, and a subjected concentrated moment. These exact solutions for the straight free surface are well known. Here, these solutions are applied to the cosine-wavy free surface problem by using the general form (28).
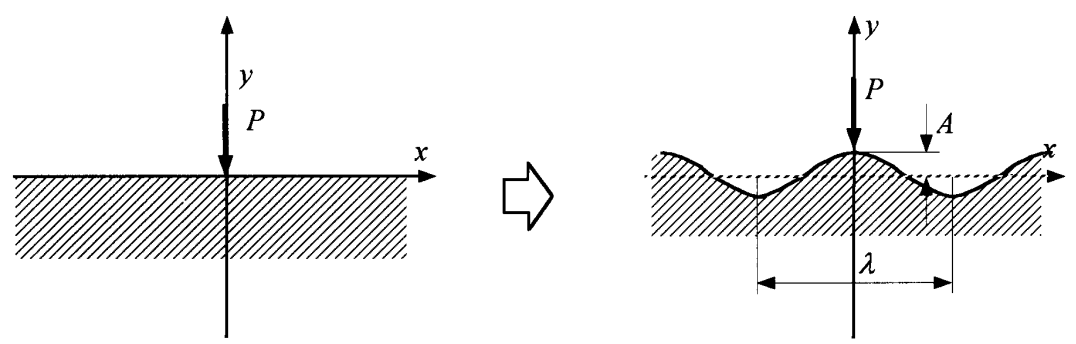

Fig.1 Semi-infinite media subjected to a point load at origin

\subsection{Semi-infinite elastic media subjected to point load}

In the problem (see Fig. 1) which semi-infinite $(y \leq 0)$ elastic media with the free surface laid along the $x$-axis is subjected to point load normal to the free surface at origin, the displacement solution is known to be expressed for plane stress,

$$
\begin{aligned}
& u=\frac{(1+v) P}{\pi E}\left[-\frac{x y}{x^{2}+y^{2}}+\left(\frac{1-v}{1+v}\right) \tan ^{-1}\left(\frac{x}{y}\right)\right] \\
& v=-\frac{(1+v) P}{\pi E}\left[\frac{y^{2}}{x^{2}+y^{2}}-\frac{1}{1+v} \log \left(x^{2}+y^{2}\right)\right]
\end{aligned}
$$

and the associated stress components are given by, 


$$
\begin{aligned}
& \sigma_{x x}=\frac{2 P}{\pi} \frac{x^{2} y}{\left(x^{2}+y^{2}\right)^{2}} \\
& \sigma_{y y}=\frac{2 P}{\pi} \frac{y^{3}}{\left(x^{2}+y^{2}\right)^{2}} \\
& \sigma_{x y}=\frac{2 P}{\pi} \frac{x y^{2}}{\left(x^{2}+y^{2}\right)^{2}}
\end{aligned}
$$

These solutions satisfy the differential equations (2) in the Cartesian coordinate system. Then, the solutions $u^{0}$ and $v^{0}$ for non-perturbation order satisfying differential equations (23) can be obtained easily by replacing $(x, y)$ with $(\xi, \eta)$. By this, equations (33) yield,

$$
\begin{aligned}
& u^{0}=\frac{(1+v) P}{\pi E}\left[-\frac{\xi \eta}{\xi^{2}+\eta^{2}}+\left(\frac{1-v}{1+v}\right) \tan ^{-1}\left(\frac{\xi}{\eta}\right)\right] \\
& v^{0}=-\frac{(1+v) P}{\pi E}\left[\frac{\eta^{2}}{\xi^{2}+\eta^{2}}-\frac{1}{1+v} \log \left(\xi^{2}+\eta^{2}\right)\right]
\end{aligned}
$$

and also equations (34) become,

$$
\begin{aligned}
& \sigma_{x x}^{0}=\frac{2 P}{\pi} \frac{\xi^{2} \eta}{\left(\xi^{2}+\eta^{2}\right)^{2}} \\
& \sigma_{y y}^{0}=\frac{2 P}{\pi} \frac{\eta^{3}}{\left(\xi^{2}+\eta^{2}\right)^{2}} \\
& \sigma_{x y}^{0}=\frac{2 P}{\pi} \frac{\xi \eta^{2}}{\left(\xi^{2}+\eta^{2}\right)^{2}}
\end{aligned}
$$

Substituting (35) and (36) into (28), the first-order solution for semi-infinite elastic media with cosine-wave surface subjected to a point load is,

$$
\begin{aligned}
& \sigma_{x x}(\xi, \eta)=\frac{2 P}{\pi}\left[\frac{\xi^{2} \eta}{\left(\xi^{2}+\eta^{2}\right)^{2}}+\frac{\xi^{2}\left(\xi^{2}-3 \eta^{2}\right)}{\left(\xi^{2}+\eta^{2}\right)^{3}} A \cos \left(\frac{2 \pi}{\lambda} \xi\right)\right] \\
& \sigma_{y y}(\xi, \eta)=\frac{2 P}{\pi}\left[\frac{\eta^{3}}{\left(\xi^{2}+\eta^{2}\right)^{2}}-\frac{\eta^{2}\left(\eta^{2}-3 \xi^{2}\right)}{\left(\xi^{2}+\eta^{2}\right)^{3}} A \cos \left(\frac{2 \pi}{\lambda} \xi\right)\right] \\
& \sigma_{x y}(\xi, \eta)=\frac{2 P}{\pi}\left[\frac{\xi \eta^{2}}{\left(\xi^{2}+\eta^{2}\right)^{2}}+\frac{2 \xi \eta\left(\xi^{2}-\eta^{2}\right)}{\left(\xi^{2}+\eta^{2}\right)^{3}} A \cos \left(\frac{2 \pi}{\lambda} \xi\right)\right]
\end{aligned}
$$

Fig. 2 shows variation of the stress component $\sigma_{y y}$ at $x=0$ and $y=-\mathrm{A}$, which takes its maximum value on the $y=$-A plane, with perturbation parameter $\varepsilon$. The solid line indicates the results obtained from the first-order solution, and circle symbol is the results by finite element analysis. Here, finite element analysis was conducted using ANSYS 5.7 with the model as shown in Fig. 3. The model was divided by structural 2-D 6-nodes triangle solid element and composed of 622 nodes and 289 elements. Boundary condition for this divided model was following; displacement components for $x$ direction at $x=0$ and $\lambda / 2$ are zero, and one for $y$ direction along the bottom of the model is also zero. Point load $-\mathrm{P} / 2$ is given at the top of cosine-wavy surface. In the comparison of the finite element analysis, the first-order solution by the perturbation theory was verified with at least ten percent accuracy. However, approaching more small perturbation parameter, the difference between the first-order and 


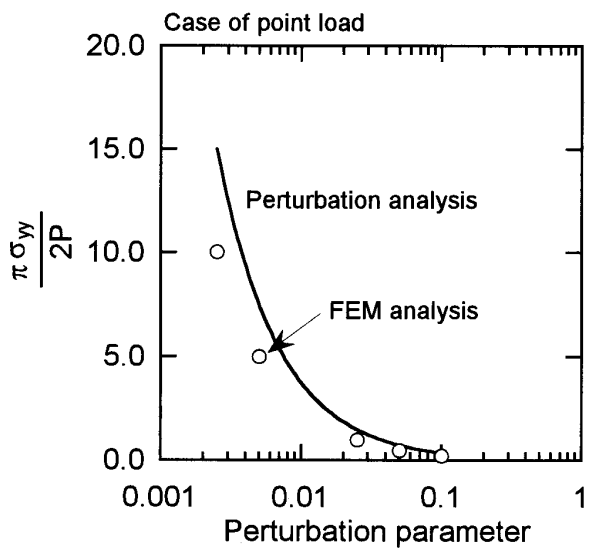

Fig.2 Variation of maximum stress component $\sigma_{y y}$ with perturbation parameter $\varepsilon$ in comparison with first-order solution (solid line) obtained in this study and finite element analysis (circle symbol).

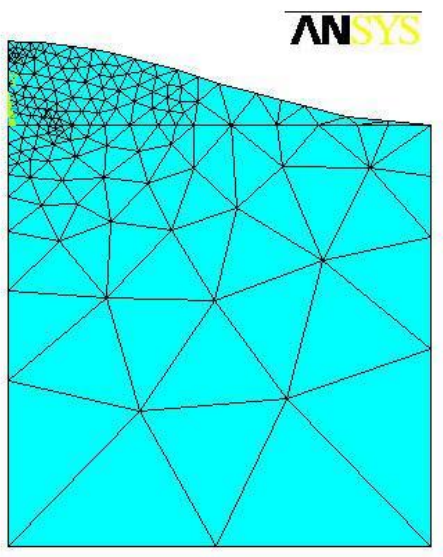

Fig.3 Finite element model employed in this study. The model was divided with structural 2-D triangle solid element. This case indicates result for $\varepsilon=0.1$

finite element solutions grows larger, becoming about twenty percent or more. This error will be improved by taking higher-order solutions.

Fig. 4 shows the distribution of the stress components along the $x$-axis at $y=-\mathrm{A}$. As the perturbation parameter increases — that is, the amplitude of the wave becomes larger - the stress distribution seems to spread along the $x$-axis and the maximum value of the stress is reduced.

\subsection{Semi-infinite elastic media subjected to concentrated moment}

In the next problem (see Fig. 5), a semi-infinite elastic media $(y \leq 0)$ with the free surface laid along the $x$-axis is subjected to a concentrated moment. The displacement solution in Cartesian coordinate system is known to be expressed as follows for plane stress,

$$
\begin{aligned}
& u=-\frac{2 M}{\pi E} \frac{\left(x^{2}-v y^{2}\right) y}{\left(x^{2}+y^{2}\right)^{2}} \\
& v=-\frac{2 M}{\pi E} \frac{\left(x^{2}+(2+v) y^{2}\right) y}{\left(x^{2}+y^{2}\right)^{2}}
\end{aligned}
$$




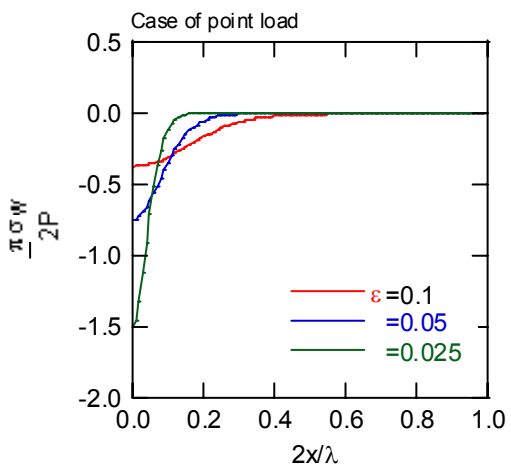

(a) Stress component $\sigma_{y y}$

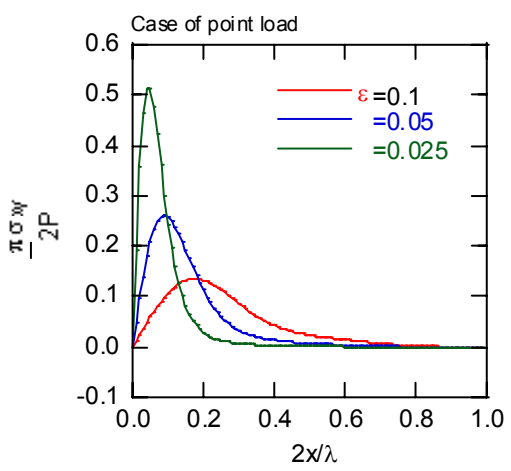

(b) Stress component $\sigma_{x y}$

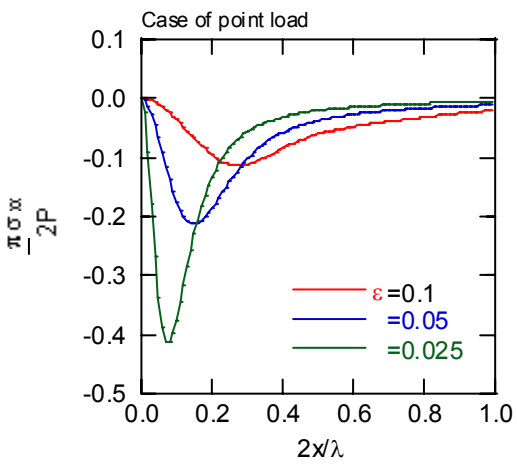

(c) Stress component $\sigma_{x x}$

Fig.4 Stress distribution along $x$-axis at $y=-A$

, and the stress components are,

$$
\begin{aligned}
& \sigma_{x x}=\frac{4 M}{\pi} \frac{x y\left(x^{2}-y^{2}\right)}{\left(x^{2}+y^{2}\right)^{3}} \\
& \sigma_{y y}=\frac{8 M}{\pi} \frac{x y^{3}}{\left(x^{2}+y^{2}\right)^{3}} \\
& \sigma_{x y}=\frac{2 M}{\pi} \frac{y^{2}\left(y^{2}-3 x^{2}\right)}{\left(x^{2}+y^{2}\right)^{3}}
\end{aligned}
$$

Using the same method used with the point load problem to obtain the first-order solution, we have, 


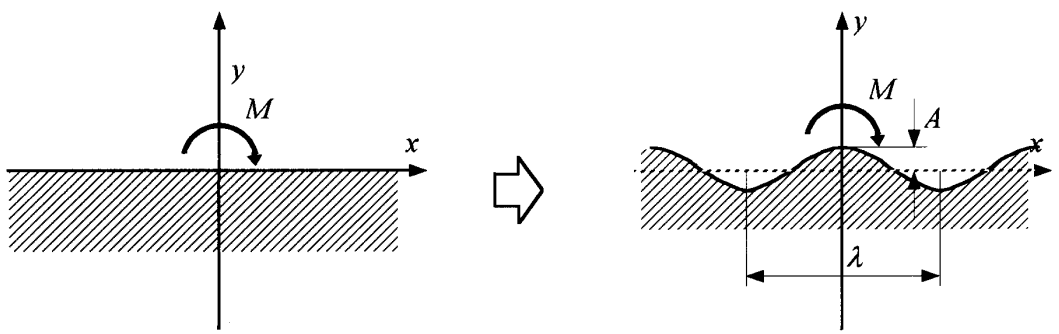

Fig.5 Semi-infinite media subjected to a concentrated moment at origin

$$
\begin{aligned}
& \sigma_{x x}=\frac{4 M}{\pi}\left[\frac{\xi \eta\left(\xi^{2}-\eta^{2}\right)}{\left(\xi^{2}+\eta^{2}\right)^{3}}+\frac{\xi^{5}-8 \xi^{3} \eta^{2}+3 \xi \eta^{4}}{\left(\xi^{2}+\eta^{2}\right)^{4}} A \cos \left(\frac{2 \pi}{\lambda} \xi\right)\right] \\
& \sigma_{y y}=\frac{8 M}{\pi}\left[\frac{\xi \eta^{3}}{\left(\xi^{2}+\eta^{2}\right)^{3}}+3 \cdot \frac{\xi \eta^{2}\left(\xi^{2}-\eta^{2}\right)}{\left(\xi^{2}+\eta^{2}\right)^{4}} A \cos \left(\frac{2 \pi}{\lambda} \xi\right)\right] \\
& \sigma_{x y}=\frac{2 M}{\pi}\left[\frac{\eta^{2}\left(\eta^{2}-3 \xi^{2}\right)}{\left(\xi^{2}+\eta^{2}\right)^{3}}+2 \cdot \frac{\eta^{5}-8 \xi^{2} \eta^{3}+3 \xi^{4} \eta}{\left(\xi^{2}+\eta^{2}\right)^{4}} A \cos \left(\frac{2 \pi}{\lambda} \xi\right)\right]
\end{aligned}
$$

Fig. 6 shows the distribution of the stress components along the $x$-axis at $y=-\mathrm{A}$. Although the stress distributions are anti-symmetric to the $y$-axis because of the anti-symmetric stressed free surface due to moment $M$, the stress distribution profiles spread with an increasing perturbation parameter, as well as the solution for the point-loaded problem.

\section{Conclusion}

The main results are;

(1) The perturbation theory was applied to a two-dimensional elastic problem with a slight-rough boundary. In this study, the equilibrium equations for displacement in Cartesian coordinates $(x, y)$ were translated into curvilinear coordinates $(\xi, \eta)$, considering the shape function $y=g(x)$ to express the rough boundary. Next, general first-order solutions for displacement and stress components were obtained through a perturbation scheme including the small parameter $\varepsilon=A / \lambda<<1$, where $A$ is amplitude and $\lambda$ is characteristic wavelength in the shape function.

(2) As applications of the general first-order solution obtained in this study, the cosine as a typical periodic function and an exponential function were considered as the shape function. The associated first-order solutions for the elastic problem with cosine and exponential wave boundary were shown.

(3) As a calculation example, the solutions for semi-infinite elastic media subjected to point load were shown, and also these were compared with the results obtained by finite element analysis. The first-order solution by the perturbation theory was verified with at least ten percent accuracy. However, approaching a smaller perturbation parameter, the difference between the first-order and finite element solutions grew larger, becoming about twenty percent or more. This error will be improved by taking higher-order solutions. 


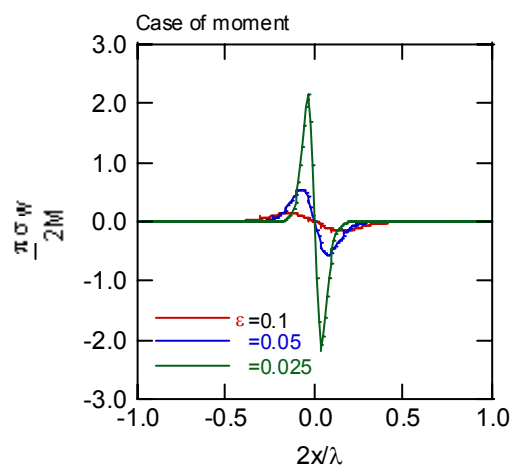

(a) Stress component $\sigma_{y y}$

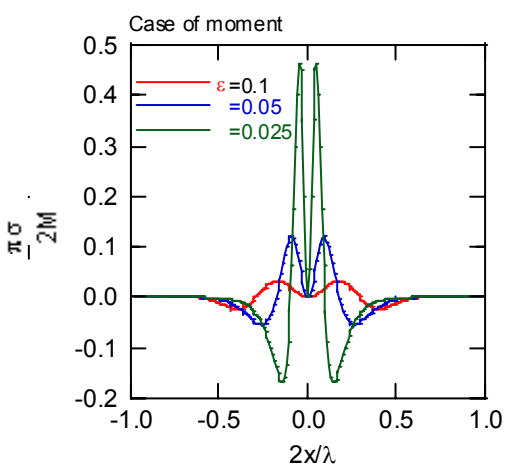

(b) Stress component $\sigma_{x y}$

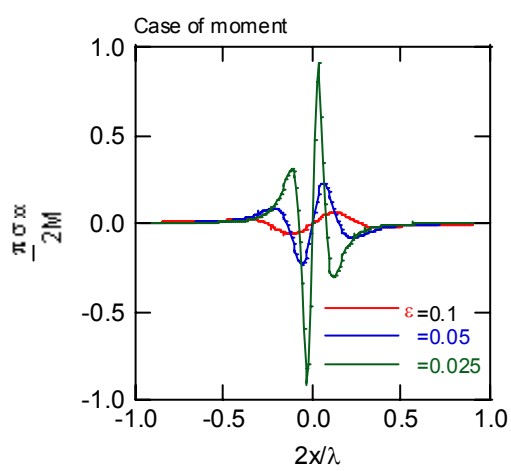

(c) Stress component $\sigma_{x x}$

Fig.6 Stress distribution along $x$-axis at $y=-A$

\section{References}

(1) S. Suresh, "Fatigue of Materials", Cambridge University Press (1998), pp.335-340.

(2) K.L. Johnson, "Contact Mechanics", Cambridge University Press (1989), pp.397-416.

(3) D.J. Strolovitz, On the stability of surfaces of stresses solids, Acta metall, Vol.37, No. 2 (1989), pp.621-625.

(4) H. Gao, Stress analysis of smooth polygonal holes via a boundary perturbation method, $J$. of Applied Mech., Vol.58 (1991), pp.851-853.

(5) J.R. Rice, First-order variation in elastic fields due to variation in location of a plannar crack front, J. of Applied Mech., Vol.52 (1985), pp.571-579.

(6) H. Gao, A boundary perturbation analysis for elastic inclusions and interfaces, Int. J. Solids Structures, Vol.28, No.6 (1991), pp. 703-725.

(7) K.S. Wang, Y.P. Yang, C.M. Chu, Stress analysis of an elastic body with a wavy surface, 
Wear, Vol.145 (1991), pp.101-112.

(8) F. Hirano, Research on stress concentration factors for two dimensional elastic bodies, Trans. Jpn. Soc. Mech. Eng., Vol.17 (1951), pp.12-16.

(9) P. Villaggio, How to model a bonded joint, J. of Applied Mech., Vol.56 (1989), pp.590-594. 